\title{
Lifshitz Transition in the Double-Core Vortex in ${ }^{3} \mathrm{He}-\mathrm{B}$
}

\author{
M. A. Silaev, ${ }^{1}$ E. V. Thuneberg, ${ }^{2}$ and M. Fogelström ${ }^{3}$ \\ ${ }^{1}$ Department of Theoretical Physics, KTH-Royal Institute of Technology, SE-10691 Stockholm, Sweden \\ ${ }^{2}$ Department of Physics, University of Oulu, FI-90014 Oulu, Finland \\ ${ }^{3}$ Department of Microtechnology and Nanoscience, Chalmers, SE-41296 Göteborg, Sweden
}

(Received 20 July 2015; published 2 December 2015)

\begin{abstract}
We study the spectrum of fermion states localized within the vortex core of a weak-coupling $p$-wave superfluid. The low energy spectrum consists of two anomalous branches that generate a large density of states at the locations of the half cores of the vortex. Fermi liquid interactions significantly stretch the vortex structure, which leads to a Lifshitz transition in the effective Fermi surface of the vortex core fermions. We apply the results to the rotational dynamics of vortices in superfluid ${ }^{3} \mathrm{He}-\mathrm{B}$ and find an explanation for the observed slow mode.
\end{abstract}

DOI: 10.1103/PhysRevLett.115.235301

PACS numbers: 67.30.he, 67.30.hj, 74.20.Rp

The double-core vortex is an amazing structure because it is the unique answer to a simple question: What is the vortex structure of a weak-coupling $p$-wave-pairing superfluid? The ground state in this case is the Balian-Werthamer (BW) state [1]. The lowest energy vortex has the doublecore structure, where the core is split into two "half cores" as depicted in Figs. 1(a) and 1(b) [2-8]. This is not only of theoretical interest since superfluid ${ }^{3} \mathrm{He}$ is close to being weak coupling, and its $B$ phase was identified as the BW state. Two vortex types have been found experimentally in ${ }^{3} \mathrm{He}-\mathrm{B}$ [9-12]. The vortex being stable in the major, lowpressure part of the phase diagram was identified as the double-core vortex. The vortex stable at higher pressures was identified as the axially symmetric $A$-phase-core vortex. Available experimental evidence is consistent with the theoretical identification of the vortex structures. In particular, the broken axial symmetry of the double-core vortex was used to explain the peculiar dynamical properties that have been observed for the low pressure vortex using the homogeneous precessing domain mode of NMR $[13,14]$. A similar double-core vortex structure has been suggested to appear in the spin-triplet heavy fermion superconductor $\mathrm{UPt}_{3}$ [15].

One of the most interesting properties of quantized vortices in superconductors and Fermi superfluids is the presence of fermionic quasiparticles localized within the vortex core at energies smaller than the bulk energy gap $[16,17]$. Generally fermionic bound states determine both the thermodynamic and dynamic properties of vortices at low temperatures [18-22]. In the rotational dynamics of the double-core vortex they are predicted to give rise to resonance absorption at the frequency comparable with the spacing of the localized energy eigenstates [23]. Recently, much attention has been focused on the topologically protected zero energy vortex-core and surface states in superfluid ${ }^{3} \mathrm{He}$ [24-27]. Particularly motivating is a predicted existence of self-conjugated Majorana states localized on half-quantum vortices in $p$-wave superfluids [28].

In this Letter we calculate the low-energy fermionic excitation spectrum of the double-core vortex. We find that the low-energy excitations mostly are localized in the two half cores. This is visualized in Figs. 1(c) and 1(d), which show the fermionic local density of states (LDOS) profiles around the vortex core. We can interpret the two half cores as potential wells for quasiparticles. The motion of the excitations between the wells depends on the potential barrier between them. We find that this barrier changes
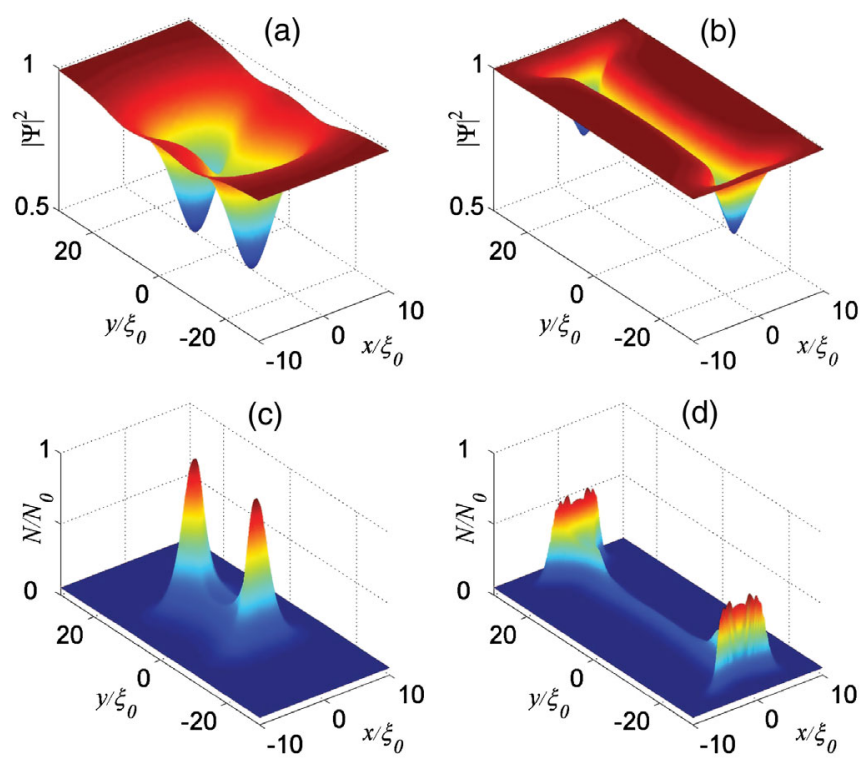

FIG. 1 (color online). (a),(b) The double-core vortex structure made visible by the pair density $|\Psi|^{2}=\sum_{\mu, i}\left|A_{\mu, i}\right|^{2}$ plotted in the $x-y$ plane perpendicular to the vortex axis at temperature (a) $T / T_{c}=0.9$ and (b) $T / T_{c}=0.1$. (c),(d) The normalized local density of states profiles demonstrating the quasiparticle wave function at the Fermi level $\varepsilon=0, \hat{p}_{z}=0$ at (c) $T / T_{c}=0.9$ and (d) $T / T_{c}=0.1$. All plots correspond to pressure $P=24$ bar. 
essentially as the distance of the wells changes as a function of pressure and temperature (see Fig. 1 to compare the vortex structures at $T / T_{c}=0.9$ and $T / T_{c}=0.1$ ). This implies a transition from excitations that circle both half cores to separate excitations that circle only a single half core. This transition can be seen as a Lifshitz-type transition in the topology of the fermionic states bound to the vortex core. We discuss how this could be observable in the time scales of rotational dynamics. Comparing our calculations with an earlier experiment reveals a serious disagreement in the model used to interpret the experimental data [14]. We construct a different model, which also provides an explanation for the long time scale observed in rotational dynamics $[13,29,30]$.

The triplet pairing of fermions in orbital $p$-wave states is described by the matrix

$$
\check{\Delta}(\boldsymbol{r}, \hat{\boldsymbol{p}})=\sum_{\alpha, i} A_{\alpha i}(\boldsymbol{r}) i \check{\boldsymbol{\sigma}}_{\alpha} \check{\sigma}_{y} \hat{p}_{i}
$$

where $\check{\sigma}_{x, y, z}$ are Pauli matrices, $\boldsymbol{p}$ is the momentum close to the Fermi surface $p \approx p_{F}=\hbar k_{F}$, and $\hat{\boldsymbol{p}}=\boldsymbol{p} / p$. The gap function $\Delta(1)$ is determined by the $3 \times 3$ order parameter matrix with complex components $A_{\alpha i}$. Here, $\alpha=x, y, z$ and $i=x, y, z$ are the spin and orbital indices, respectively.

In the weak coupling theory of a $p$-wave superfluid, the stable state has the BW form [1]. In the BW state, the order parameter far from the vortex axis is $A_{\alpha i}=\Delta_{0} \exp (i \varphi) R_{\alpha i}$. Here, $\varphi$ is the azimuth with respect to the vortex axis, $R_{\alpha i}$ is a constant rotation matrix, and $\Delta_{0}$ is the order parameter amplitude. Near the vortex axis a more sophisticated structure appears [2-8]. It is energetically favorable to change the sign of the order parameter across the vortex axis by spin rotation of the BW-state matrix $A_{\alpha i}$ by $\pi$ [4]. This effectively results in splitting of a singly quantized vortex to a pair of half-quantum vortices that are bound together by a planar-phase domain wall. For illustration see Figs. 1(a) and 1(b), which show the pair density $|\Psi|^{2}=$ $\sum_{\alpha, i}\left|A_{\alpha i}\right|^{2}$ in the $x-y$ plane. The pair density has two distinct minima, whence the name double-core vortex.

To determine the vortex structure we calculate selfconsistently the order parameter and the Fermi-liquid self-energy [31]. The numerics is performed as described in Ref. [6], i.e., using the explosion trick to solve the Eilenberger transport equation. We extend the previous work [6,7] to higher accuracy, lower temperatures, and different values of the Fermi-liquid parameter $F_{1}^{s}$ corresponding to different pressures. The parameter $F_{1}^{s}$ determines the feedback of the superfluid mass current on the order parameter and can significantly change both the vortex structure and the spectrum of bound fermions.

The distance $a$ between the half cores is shown Fig. 2. Its scale is $R_{0}=\left(1+F_{1}^{s} / 3\right) \xi_{0}$, where $\xi_{0}=\hbar v_{F} / 2 \pi T_{c}$ is the coherence length and $v_{F}$ is the Fermi velocity. As $F_{1}^{\mathrm{s}}$ in liquid ${ }^{3} \mathrm{He}$ ranges from 5.4 to 14.6 depending on pressure $P$

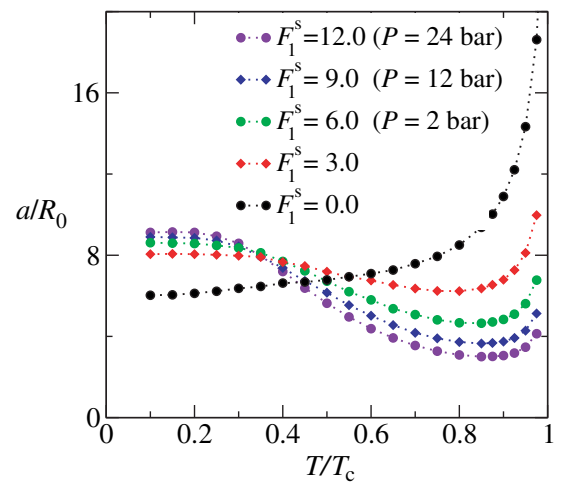

FIG. 2 (color online). Distance $a$ between the half cores in the double-core vortex at different values of the Fermi liquid parameter $F_{1}^{s}$. The locations of the half cores are determined from zeros of the supercurrent density.

[32], the two length scales can differ essentially. Thus, at large values of $F_{1}^{\mathrm{s}}$, corresponding to high pressures, the vortex size at a low temperature is much larger than the coherence length. For example, $a=46 \xi_{0}$ in the case of Fig. 1(b). Figure 2 also shows strong temperature and pressure dependence. The distance of the half cores grows almost threefold when the temperature decreases from $0.9 T_{c}$ to $0.1 T_{c}$ at 24 bar. Similarly, as a function of pressure the distance $a$, measured in units of $\xi_{0}$, grows almost twofold when the pressure increases from 0 to 24 bar at a low temperature.

We calculate the excitation spectrum of the double-core vortex using the self-consistent order parameter field. This is done by solving the eigenvalue problem for the system of Andreev equations, which are ordinary differential equations describing the propagation of a quasiparticle wave function along classical trajectories.

The momentum $\boldsymbol{p}$ of a low energy excitation is close to the Fermi surface, $p \approx p_{F}$. The classical trajectories are straight lines parallel to $p$. In studying a vortex we fix the $z$ axis as the vortex axis, and we parametrize the momentum direction $\hat{\boldsymbol{p}}=\left(\hat{p}_{\perp} \cos \theta_{p}, \hat{p}_{\perp} \sin \theta_{p}, \hat{p}_{z}\right)$. The direction on the trajectory is fixed by giving $\hat{p}_{z}$ and $\theta_{p}$. The location of the trajectory is given by the impact parameter $b$, the coordinate measuring the distance from the vortex axis. The parametrization is visualized in Fig. 3(a). The impact parameter is related to the projection of the angular momentum $\mu$ on the vortex axis through the usual classical mechanics formula $\mu=p_{\perp} b$. The quasiclassical energy spectrum is given by $\varepsilon=\varepsilon_{i}\left(\hat{p}_{z}, \theta_{p}, b\right)$, where the parameters $\hat{p}_{z}, \theta_{p}, b$ specify the classical trajectory and integer $i$ counts the eigenvalues of the Andreev equations on a given trajectory [33]. Figure 3(b) shows a bunch of trajectories at the Fermi level and $\hat{p}_{z}=0$. The concentration of the trajectories at the two half cores results in the large LDOS at the half cores. The concave triangular shape of the caustic of the trajectories at the half cores is clearly visible in the LDOS shown in Fig. 1(d). Also the classically 
(a)

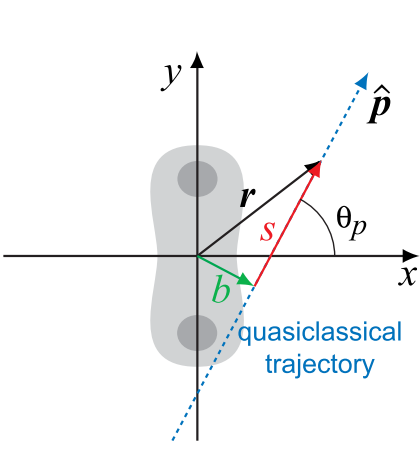

(c)

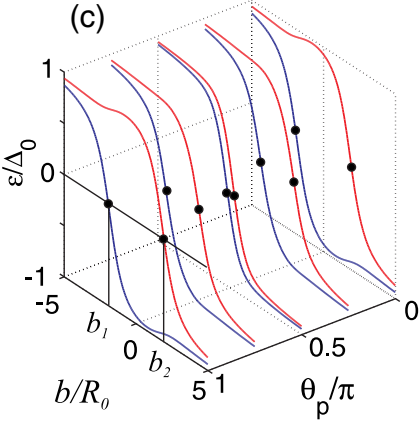

(b)

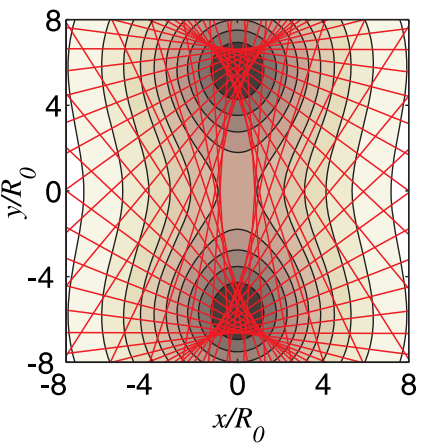

(d)

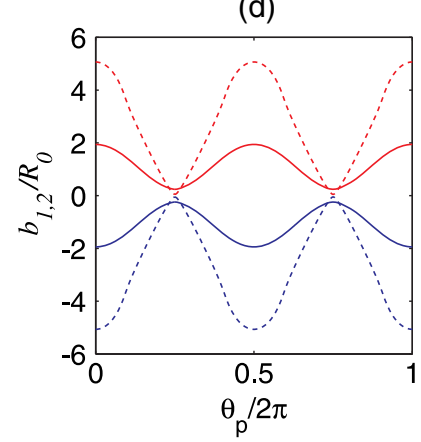

FIG. 3 (color online). (a) Schematic plot of a quasiclassical trajectory in the $x-y$ plane in the direction of $\left(\cos \theta_{p}, \sin \theta_{p}\right)$ passing the vortex axis at distance $b$ (impact parameter). A point $\boldsymbol{r}=(x, y)$ on the trajectory is determined by the coordinate $s$. (b) A bunch of quasiparticle trajectories (straight lines) at the Fermi level $\varepsilon=0$ and $\hat{p}_{z}=0$ superimposed on the pair-density contour plot at $T / T_{c}=0.5$ and $F_{1}^{s}=0$. (c) Anomalous branches of the quasiparticle spectrum $\varepsilon=\varepsilon_{1,2}\left(b, \theta_{p}\right)$ at $\hat{p}_{z}=0, F_{1}^{s}=12$, and $T=0.9 T_{c}$. (d) Two sheets of the effective Fermi surface $b=b_{1,2}\left(\theta_{p}\right)$ at $\hat{p}_{z}=0, F_{1}^{s}=12, T=0.9 T_{c}$ (solid lines) and $T=0.1 T_{c}$ (dashed lines).

nonallowed region around the vortex axis in Fig. 3(b) can be recognized in Fig. 1(c) as a valley in the LDOS profile in the region between the half cores.

Because of the lifted spin degeneracy, singly quantized vortices in ${ }^{3} \mathrm{He}-\mathrm{B}$ have two anomalous branches of the quasiparticle spectrum [34]. At low energy compared to the bulk energy gap, $|\varepsilon| \ll \Delta_{0}$, they can be represented as

$$
\varepsilon_{i}\left(\hat{p}_{z}, \theta_{p}, b\right)=-\omega_{i} p_{F}\left(b-b_{i}\right)
$$

where $i=1,2$. Here, $b_{i}\left(\hat{p}_{z}, \theta_{p}\right)$ is the impact parameter that corresponds to vanishing excitation energy, and $\omega_{i}\left(\hat{p}_{z}, \theta_{p}\right)$ indicates the slope of the energy at $b=b_{i}$. The new feature in a nonaxisymmetric vortex is that these parameters depend on the trajectory direction $\theta_{p}$ in the $x-y$ plane.

Figure 3(c) shows the calculated quasiparticle energies as a function of impact parameter $b$ and different directions of the trajectory $\theta_{p}$. The curves cross the Fermi level at a finite $b$ in accordance with Eq. (2). These locations in the

energy spectrum $b_{1,2}\left(\theta_{p}\right)$ are shown by the black dots in Fig. 3(c). The states at the Fermi level in the spectrum (2) form a $2 \mathrm{D}$ effective Fermi surface $b=b_{1,2}\left(\hat{p}_{z}, \theta_{p}\right)$ in the 3D space formed by the quasiclassical quantum numbers $\left(\hat{p}_{z}, \theta_{p}, b\right)$ in the vortex core. Because of the two nondegenerate branches (2), there are two sheets in the Fermi surface. One more representation of this is given in Fig. 3(d). It shows $b_{1,2}$ as a function of $\theta_{p}$. The curves depend also on $\hat{p}_{z}$ but that dependence is less important in the following because $\hat{p}_{z}$ is conserved. For comparison, the trajectories passing precisely through the half cores at $y=$ $\pm a / 2$ would correspond to curves $b\left(\theta_{p}\right)=\mp \frac{1}{2} a \cos \theta_{p}$.

The topology of the effective Fermi surface is determined by the behavior of the zero energy lines $b_{1,2}\left(\theta_{p}\right)$ at $\theta_{p}=\pi(n+1 / 2)$ with $n=1$, 2. At these angles the quasiparticle trajectories pass through both half cores. In general there is overlap of the quasiparticle wave functions localized at different half cores. This results in no sign change of $b_{1,2}\left(\theta_{b}\right)$. That is, there is anticrossing of the two branches and a finite splitting $2 \delta b=\left|b_{1}-b_{2}\right|>0$ at $\theta_{p}=\pi / 2$, as shown by the solid lines in Fig. 3(d). Physically, this means that an excitation created at one half core will jump periodically between the half cores.

The growing core separation (compared to $\xi_{0}$ ) at low temperatures and large pressures reduces the overlap of the quasiparticle wave functions located at different half cores. As a result the splitting $2 \delta b$ becomes extremely small as shown by the dashed lines in Fig. 3(d) for $F_{1}^{s}=12$ and $T=0.1 T_{c}$. In this case Landau-Zener (LZ) tunneling between the quasiclassical branches (2) becomes important. The probability $W$ of these transitions can be found from the conventional approach $[35,36]$ by taking $\theta_{p}$ and the angular momentum $\mu=p_{\perp} b$ as the conjugate variables. Near the anticrossing point at $\theta_{p}=\pi / 2$ we can approximate $b_{1,2}\left(\theta_{p}\right) \approx \pm \sqrt{\delta b^{2}+(a \theta / 2)^{2}}$, where $\theta=\theta_{p}-\pi / 2$. The transition probability is given by $W=\exp \left[-2 k_{\perp} \operatorname{Im} \int_{0}^{i \theta^{*}}\left(b_{1}-b_{2}\right) d \theta\right]$, where $i \theta^{*}=2 i \delta b / a$ is the intersection point of the quasiclassical branches in the complex plane. A simple calculation yields $W=$ $\exp \left[-2 \pi \hat{p}_{\perp}(\delta b / \Delta b)^{2}\right]$, where $\Delta b=\sqrt{a / k_{F}}$ has the physical meaning of the quantum mechanical uncertainty of the impact parameter.

Once the transition probability becomes large, $W \approx 1$, the LZ tunneling changes the topology of the effective Fermi surface so that the quasiparticles remain localized in one or the other of the half cores. In Fig. 3(d) this means a transition to the intersecting zero energy curves $\tilde{b}_{1(2)}\left(\theta_{p}\right)=$ $b_{1(2)}\left(\theta_{p}\right)$ for $-\pi / 2<\theta_{p}<\pi / 2$ and $\tilde{b}_{1(2)}\left(\theta_{p}\right)=b_{2(1)}\left(\theta_{p}\right)$ for $\pi / 2<\theta_{p}<3 \pi / 2$. The calculated LZ probability $W\left(T, P, \hat{p}_{z}\right)$ is shown in the Supplemental Material [37] to demonstrate that the condition $W \approx 1$ is realized in the double-core vortex at large pressures and low temperatures. The crossover from the split $b_{1,2}\left(\theta_{p}\right)$ to the intersecting isoenergetic lines $\tilde{b}_{1,2}\left(\theta_{p}\right)$ is an analog of the Lifshitz transition [39] changing the topology of the Fermi surface. 
TABLE I. Values of the minigap $E_{m} / h(\mathrm{kHz})$ at different pressures and temperatures.

\begin{tabular}{lccc}
\hline \hline & $P=2$ bar & $P=12$ bar & $P=24$ bar \\
\hline$T=0.05 T_{c}$ & 27 & 71 & 98 \\
$T=0.5 T_{c}$ & 22 & 65 & 106 \\
\hline \hline
\end{tabular}

The transition leads to a formation of two spatially separated low-energy fermionic states localized at the half cores. Whether there are Majorana states precisely at the Fermi level [28] or not [27] is beyond our quasiclassical approach. The transition affects the rotational dynamics of the double-core vortex. The bound fermions in the core respond to oscillation of the core orientation. A friction torque acting on a rotating vortex core can be expressed by a friction coefficient $f=f_{1} p_{F}\left(k_{F} \xi_{0}\right)^{2}$, where $f_{1} \sim 1$ is dimensionless and the factor $p_{F}\left(k_{F} \xi_{0}\right)^{2}$ is determined by the density of quasiparticles in the vortex core. The expression for the friction torque [23] yields resonance peaks in $f$ located at angular frequencies $\omega \approx n E_{m} / \hbar$, where $n$ is integer. Here, $E_{m}$ is the spacing of the quantized energy levels obtained from the quasiclassical spectrum (2) using the Bohr-Sommerfeld quantization rule for the angular momentum [23], $E_{m}=\hbar\left\langle\omega_{1}^{-1}\left(\hat{p}_{z}=0\right)\right\rangle^{-1}$, where $\langle\cdots\rangle$ denotes the average over $\theta_{p}$. The scale of the minigap is determined by $\hbar / \tau_{n}=\left(2 \pi T_{c}\right)^{2} / v_{F} p_{F}$, which is on the order of the quasiparticle relaxation rate in the normal state. The calculated values of the minigap are listed in Table I.

The amplitudes of the resonances are determined by the Fourier amplitudes of the zero-energy curves shown in Fig. 3, $A_{n} \sim\left|\int d \theta_{p} e^{i n \theta_{p}} b_{1}\left(\theta_{p}\right)\right|$. From the plots in Fig. 3(d) one can see that at pressures below the Lifshitz transition the largest components are those with double frequency $\hbar \omega=2 E_{m}$. At pressures above the transition the amplitudes are determined by the harmonics of the intersecting curves $\tilde{b}_{i}\left(\theta_{p}\right)$, which have the strongest matrix element at $\hbar \omega=E_{m}$. The difference in the friction coefficient $f_{1}$ in the two cases is demonstrated in Fig. 4 for different values of the quasiparticle relaxation time $\tau$. In the following we show that at least the low frequency limit of the curves in Fig. 4 is experimentally accessible.

Kondo et al. $[13,14]$ have studied a sample of rotating ${ }^{3} \mathrm{He}-\mathrm{B}$ using the homogeneously precessing domain. In this mode the magnetization $\boldsymbol{M}$ is tipped by a large angle $\left(>104^{\circ}\right)$ from the field direction $\boldsymbol{B}$. It was found that the contribution of vortices to the relaxation changed on a few minute time scale [13]. The interpretation was that the double-core vortex gets twisted as its end points (at $z= \pm L / 2$ ) are pinned but in the bulk the rotating magnetization exerts a torque on the core. A quantitative model was constructed for the vortex core rotation angle $\phi(t, z)$ as a function of time $t$ and $z$. The parameters of the model were determined by fitting to the experiment [14]. These include the friction parameter $f$, the dipole torque $T_{D}$, which drives the vortex in the presence of rotating (a)

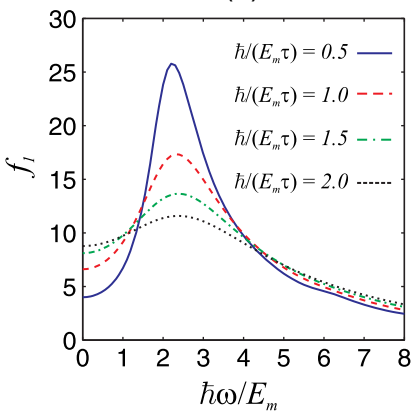

(b)

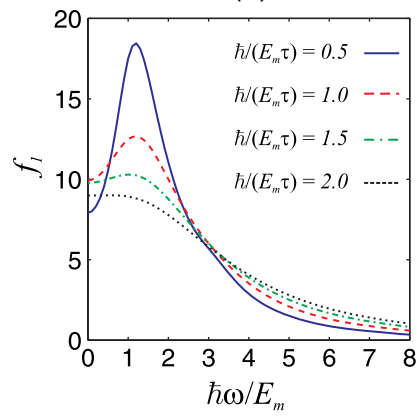

FIG. 4 (color online). Demonstration of the effect of the Lifshitz transition on the rotational friction coefficient $f_{1}$ plotted as a function of frequency $\omega$ for (a) $W=0$ and (b) $W=1$. The vortex structure and the excitation spectrum are calculated at $T=0.5 T_{c}$ and (a) $P=2$ bar and (b) $P=24$ bar. The minigap values are given in Table I. According to mutual friction measurements [12] $\hbar / E_{m} \tau=0.7$ at $P=24$ bar but a wider range is given to illustrate the influence of relaxation on the shape of the absorption peak.

magnetization, and the rigidity $K$, which gives the energy caused by twisting the core, $F_{\text {twist }}=\frac{1}{2} K\left(\partial_{z} \phi\right)^{2}$.

By precise calculation of the vortex structure we can now calculate the vortex parameters. We find a value of $f$ that is 3 orders of magnitude larger than that fitted by Kondo et al. [14]. Thus, a serious revision of the model has to be made. The large value of $f$ means that only a negligible fraction of energy dissipation comes from vortex core rotation. Thus, essentially all dissipation has to arise from normalsuperfluid disequilibrium [40], spin diffusion, and radiation of spin waves. Without going into details, these can be incorporated by allowing an elastic vortex structure, where the rotation angle $\alpha(t, z)$ at a distance from the vortex axis (where the dipole torque acts) can be different from the vortex core angle $\phi(t, z)$. These are bound by elasticity energy $\frac{1}{2} T_{A}(\alpha-\phi)^{2}$, and both angles have their own friction coefficients: $f \dot{\phi}=-\delta F / \delta \phi, g \dot{\alpha}=-\delta F / \delta \alpha$. This model results in the diffusion equation [37]

$$
\dot{\phi}=\frac{K}{f} \partial_{z}^{2} \phi+\frac{P_{g}}{\omega f},
$$

where $P_{g}$ is the power absorption per vortex length. An important virtue of this model is that based on our calculations of $f$ and $K$, Eq. (3) predicts a time scale $L^{2} f / \pi^{2} K$ of several minutes. Thus, Eq. (3) gives a simple explanation for the observed slow mode $[13,29,30]$, which remained unexplained in previous models [14,30].

In summary, we have investigated the spectrum of bound fermion states localized within the vortex core of a weakcoupling $p$-wave superfluid. We predicted a Lifshitz transition, which separates low-energy quasiparticle states at the half cores and affects the rotational dynamics. Applying our results to Ref. [14] explains the observed 
long time scale and thus gives one more piece of evidence of the double-core nature of the low pressure vortex in ${ }^{3} \mathrm{He}-\mathrm{B}$.

We thank I. Khaymovich, N. Kopnin, A. Mel'nikov, and G. Volovik for useful discussions. This work was supported by the Academy of Finland and the Tauno Tönning Foundation. M. A. S. and M. F. acknowledge support from the Swedish Research Council (VR).

[1] R. Balian and N. R. Werthamer, Phys. Rev. 131, 1553 (1963).

[2] E. V. Thuneberg, Phys. Rev. Lett. 56, 359 (1986).

[3] M. M. Salomaa and G. E. Volovik, Phys. Rev. Lett. 56, 363 (1986).

[4] E. V. Thuneberg, Phys. Rev. B 36, 3583 (1987).

[5] G. E. Volovik, Pis'ma Zh. Eksp. Teor. Fiz. 52, 972 (1990) [JETP Lett. 52, 358 (1990)].

[6] M. Fogelström and J. Kurkijärvi, J. Low Temp. Phys. 98, 195 (1995); 100, 597(E) (1995).

[7] M. Fogelström and J. Kurkijärvi, J. Low Temp. Phys. 116, 1 (1999).

[8] T. Kita, Phys. Rev. B 66, 224515 (2002).

[9] O. T. Ikkala, G. E. Volovik, P. J. Hakonen, Yu. M. Bun'kov, S. T. Islander, and G. A. Kharadze, Pis'ma Zh. Exp. Teor. Fiz. 35, 338 (1982) [JETP Lett. 35, 416 (1982)].

[10] P. J. Hakonen, M. Krusius, M. M. Salomaa, J. T. Simola, Yu. M. Bunkov, V. P. Mineev, and G. E. Volovik, Phys. Rev. Lett. 51, 1362 (1983).

[11] J. P. Pekola, J. T. Simola, P. J. Hakonen, M. Krusius, O. V. Lounasmaa, K. K. Nummila, G. Mamniashvili, R. E. Packard, and G. E. Volovik, Phys. Rev. Lett. 53, 584 (1984).

[12] T. D. C. Bevan, A. J. Manninen, J. B. Cook, H. Alles, J. R. Hook, and H. E. Hall, J. Low Temp. Phys. 109, 423 (1997).

[13] V. V. Dmitriev, Y. Kondo, J. S. Korhonen, M. Krusius, Yu. M. Mukharskiy, E. B. Sonin, and G. E. Volovik, Physica (Amsterdam) 165B-166B, 655 (1990).

[14] Y. Kondo, J. S. Korhonen, M. Krusius, V. V. Dmitriev, Y. M. Mukharsky, E. B. Sonin, and G. E. Volovik, Phys. Rev. Lett. 67, 81 (1991).

[15] Y. Tsutsumi, K. Machida, T. Ohmi, and M. Ozaki, J. Phys. Soc. Jpn. 81, 074717 (2012).
[16] C. Caroli, P. G. de Gennes, and J. Matricon, Phys. Lett. 9, 307 (1964).

[17] J. Bardeen, R. Kümmel, A. E. Jacobs, and L. Tewordt, Phys. Rev. 187, 556 (1969).

[18] A. I. Larkin and Yu. N. Ovchinnikov, Phys. Rev. B 57, 5457 (1998).

[19] D. Rainer, J. A. Sauls, and D. Waxman, Phys. Rev. B 54, 10094 (1996).

[20] M. Stone, Phys. Rev. B 54, 13222 (1996).

[21] N. B. Kopnin, Rep. Prog. Phys. 65, 1633 (2002).

[22] N. Nakai, P. Miranovic, M. Ichioka, and K. Machida, Phys. Rev. B 73, 172501 (2006).

[23] N. B. Kopnin and G. E. Volovik, Phys. Rev. B 57, 8526 (1998).

[24] M. A. Silaev and G. E. Volovik, J. Low Temp. Phys. 161, 460 (2010).

[25] M. A. Silaev and G. E. Volovik, Zh. Exp. Teor. Fiz. 146, 1192 (2014) [Sov. Phys. JETP 119, 1042 (2014).]

[26] T. Mizushima, Y. Tsutsumi, M. Sato, and K. Machida, J. Phys. Condens. Matter 27, 113203 (2015).

[27] Y. Tsutsumi, T. Kawakami, K. Shiozaki, M. Sato, and K. Machida, Phys. Rev. B 91, 144504 (2015).

[28] D. A. Ivanov, Phys. Rev. Lett. 86, 268 (2001).

[29] E. B. Sonin, Y. Kondo, J. S. Korhonen, and M. Krusius, Europhys. Lett. 22, 125 (1993).

[30] M. Krusius, J. S. Korhonen, Y. Kondo, and E. B. Sonin, Phys. Rev. B 47, 15113 (1993).

[31] J. W. Serene and D. Rainer, Phys. Rep. 101, 221 (1983).

[32] D. S. Greywall, Phys. Rev. B 33, 7520 (1986).

[33] A. F. Andreev, Zh. Exp. Teor. Fiz. 46, 1823 (1964) [Sov. Phys. JETP 19, 1228 (1964)]

[34] M. A. Silaev, Pis'ma Zh. Exp. Teor. Fiz. 90, 433 (2009) [JETP Lett. 90, 391 (2009)].

[35] L. D. Landau and E. M. Lifshitz, Quantum Mechanics (Pergamon Press, New York, 1965).

[36] A. S. Mel'nikov, D. A. Ryzhov, and M. A. Silaev, Phys. Rev. B 78, 064513 (2008).

[37] See Supplemental Material at http://link.aps.org/ supplemental/10.1103/PhysRevLett.115.235301, which includes Ref. [38], for plots of LZ tunneling probability, details of rotational dynamics and calculation of parameters.

[38] E. V. Thuneberg, J. Low Temp. Phys. 122, 657 (2001).

[39] I. M. Lifshitz, Zh. Exp. Teor. Fiz. 38, 1569 (1960) [Sov. Phys. JETP 11, 1130 (1960)].

[40] A. J. Leggett and S. Takagi, Ann. Phys. (N.Y.) 106, 79 (1977). 